\title{
Masquelet Induced Membrane Technique for The Surgical Treatment of Large Bone Defects: The Reasons for Successes and Failures
}

\author{
Marjorie Durand $^{1 *}$, Jean-Marc Collombet ${ }^{1}$ and Laurent Mathieu ${ }^{2}$ \\ ${ }^{1}$ Department of the Forces Medical and Surgical Support (SMCF), France \\ ${ }^{2}$ Service of Chirurgie Orthopedic, Percy Military Hospital, France
}

*Corresponding author: Marjorie Durand, Biomedical Research Institute of Tte Armed Forces (IRBA) Département Of Forces Medical and Surgical Support (SMCF), 1 Place Général Valérie Andre, BP 7391223 Brétigny Sur Orge Cedex, France.

To Cite This Article: Marjorie Durand. Masquelet Induced Membrane Technique for The Surgical Treatment of Large Bone Defects: The Reasons for Successes and Failures. Am J Biomed Sci \& Res. 2019 - 2(4). AJBSR.MS.ID.000600. DOI: 10.34297/AJBSR.2019.02.000600

Received: April 13, 2019 | Published: April 23, 2019

Keywords: Masquelet Induced-Membrane Technique; Bone Repair

Abbrevations: RIA: Reamer-Irrigator-Aspirator; IMT: Induced-Membrane Technique; IM: Induced Membrane; PMMA: Polymethylmethacrylate; ABG: Autologous Bone Graft; ICBG: Iliac Crest Bone Graft; MMP: Matrix Metalloproteinase; VEGF: Vascular Endothelial Growth Factor; TGF- $\beta 1$ :

Transforming Growth Factor- $\beta 1$; BMP-2: Bone Morphogenic Protein-2; vWF: von Willerbrand Factor; IGF-1: Insulin-like Growth Factor-1

\section{Introduction}

Segmental bone loss can result from high-energy trauma, infection, tumor resection or revision surgery. For the reconstruction of small defects $(<5 \mathrm{~cm})$, autologous bone grafts remain the standard of care [1]. Bone graft harvesting is generally performed in the iliac crest where up to $40 \mathrm{ml}$ of graft material can be collected [2]. Another bone graft source is the reamerirrigator-aspirator (RIA) procedure consisting in a simultaneous reaming of the medullary canal of the long bones and a combined irrigation- aspiration system to collect bone marrow and bony fragments into a closed suction bag (up to $90 \mathrm{ml}$ of graft material) [3]. However, for bone defects more than $5 \mathrm{~cm}$, the application of only autologous bone is suboptimal. Indeed, the grafting material is unable to successfully incorporate into the defect, thus leading to the clearance of the graft [4].

Different surgical strategies have been developed to address this challenge, including the Masquelet induced-membrane technique (IMT). This surgical approach based on the induction of a foreign-body granulation membrane has revolutionized the management of large bone defects. From its introduction in the late eighties to nowadays, still little is known about the mechanism of action of the induced membrane and even more importantly why sometimes IMT fails. At the crossroads of medicine and biology, this review collects information about the reasons for successes and failures of the IMT, thus providing recommendations about this surgical procedure.
The IMT, first described by Pr Alain Charles Masquelet in 1986, is a two-stage surgery [5]. In the first step, a radical debridement of bone and soft tissue is performed followed by the implantation of polymethylmethacrylate (PMMA) cement spacer into the bone defect. The second stage of the surgery is usually performed 6 to 8 weeks later, when a membrane called "induced-membrane" (IM) encapsulates the foreign-body PMMA cement spacer. Then, the IM is opened, and the cement spacer is removed carefully in order not to alter the IM. Then, the resulting cavity is filled with grafting material, which is iliac crest bone or RIA-collected autograft.

Presence of the IM at the lesion site allows reconstruction of massive bone defects (up to $25 \mathrm{~cm}$ ), and bone union time is independent of the bone defect size [5]. Moreover, the IMT is also clinically effective even if the recipient site has been irradiated or infected [6,7]. So, how does the IM contribute to bone repair? Firstly, the IM acts like a physical barrier between the defect and the surrounding soft tissues, thus preventing the invasion of fibrous tissue within the defect area [8]. Apart from this physical role, there are also indications that the IM actively contributes to the healing process by creating a sealed biologically privileged-chamber at the defect site.

By using an IMT rabbit model Pélissier et al. were the first to report the secretion by the IM of key growth factors including VEGF (vascular endothelial growth factor), TGF- $\beta 1$ (transforming growth 
factor- $\beta 1$ ) and BMP-2 (bone morphogenic protein-2) improving both angiogenesis and osteogenesis [9]. A substantial number of animal and translational studies later confirmed the expression of these factors and others, including the angiogenic von Willerbrand factor (vWF) [10], CD31 [11], Interleukin-6 and Interleukin-8 proinflammatory cytokines [10].

From a cellular perspective, it is now clear that IM is a vascularized collagen-based matrix entrapping immune cells (macrophages, lymphocytes) [12,13], as well as bone remodeling osteoclasts [14] and osteoprogenitor cells (mesenchymal stromal cells) [13]. Considering the well-characterized biological properties of the IM, a new research area aiming to enhance the privileged environment of the IM tissue bed recently arises from literature. Different strategies are tested. Among them, a very simple approach consisting in IM scrapping during the second stage of the surgery was described by Luangphakdy in a goat model of Masquelet surgery [15]. Promising results showing a 2-fold increase in regenerated bone volume need to be confirmed clinically.

If there is increasing biological evidences to explain the IM positive influence on bone repair as described above, only sparse prospective or retrospective studies focused on the therapeutic failures related to the Masquelet technique [16-22]. Among them, 2 major reports reviewed the success/failure of the IMT performed in 69 children [18] and 427 adult [19] patients to address critical size bone defects. In children, the mean bone union rate after a single IMT was $58 \%$ and in $42 \%$ children, various complications including non-union (23\%), graft resorption or fracture $(9 \%$ for each) and deep infection (only 1.5\%) requiring revision surgeries. Following these re-interventions, bone union mean rate raised up to $87 \%$. Interestingly, however without any quantitative statistical meta-analysis due high data variability, the authors suspected some failure risk factors, namely children sex (boy), bone defect origin and size (malignant tumors and larger bone resection), bone injury location (femur), unstable osteosynthesis fixation, mixture of the bone graft with additional bone substitute as well as a longer time between the two IM surgical steps. In adults, complications encountered during IMT reached $49.6 \%$ and the global bone union mean rate after revision surgeries (in 18\% of complication cases) was $89.7 \%$ which are quite like the results obtained in children population.

In terms of complication origins and related bone nonunions, Morelli et al. [19] highlighted the involvement of sporadic superficial and deep bacterial infections as well as persistent infections, fixation material failure, misalignment and re- fractures. Despite the huge number of considered patients, due to data heterogeneity, meta-analysis only evidenced a positive correlation between the need of iterative surgeries and lack of bone union as well as complications. There was also a significant correlation when comparing occurrence of surgical complications with detected bone infection during IM procedure. Another interesting feature of this study lied in the direct complication (mainly durable donor site pain, infection, hypertrophic scars and possibly donor bone fractures) rates in the donor site after bone graft harvesting which was only $6 \%$ using the RIA technique while reaching $19.4 \%$ by performing iliac crest harvesting.
Three patient database reviews $[2,3,23]$ compared RIA technique versus iliac crest harvesting in terms of donor site morbidity, graft quality and bone repair efficiency in the context of gold-standard autologous bone graft (ABG) and/or Masquelet technique. To summarize these data, RIA group harbored lower long-term pain scores [2], higher volume of harvested graft (up to $90 \mathrm{~mL}$ ), lower local infection and complication rates at donor sites $[3,23]$ as compared to iliac crest bone graft -ICBG) group. To the opposite, the ICBG group exhibited about twice less blood loss during ABG surgery than the RIA group [23]. Nevertheless, there was no difference between RIA and ICBG groups to achieve bone union (in $90 \%$ and $80 \%$ of cases; respectively) [2]. These considerations must be considered to choose the bone graft collection procedure for the second IMT step.

Several other prospective/retrospective studies performed on smaller cohorts however presenting more homogenous data provided promising clues to explain complications and failures in IMT. In a small cohort ( $\mathrm{n}=17$ patients), El-Alfy \& Ali [16] observed an IMT failure rate of $17.6 \%$ and confirmed that failures originated from graft lysis, infection persistence and refracture. Both Azi et al. [17] and Giotikas et al. [22] pointed out that infection persistence and graft resorption were the major causes for Masquelet technique complications and could lead to up 36\% mean rate IMT failures.

In an elegant review, Giannoudis et al. [20] indicated that both staphylococcous aureus and coagulase negative staphylococcous were generally responsible for complications and they gave some detailed strategies on the way they proceeded to overcome IMT failures in their large patient cohort ( $n=43$ patients). Recently, Siboni et al. [21] mentioned that smoking could alter bone union in Masquelet technique. This is in agreement with Scolaro et al. [24] showing in a database review that smoking had a negative influence on open or tibial fracture repair and a decreased complication rate was observed in patients who stop smoking before surgery. This could may be explaining the important rate of patient exhibiting bone non- unions after ABG using RIA technique (35.6\%) as evoked by Mets makers et al. [25] since $65.3 \%$ of the patients $(n=72)$ were smokers. However, statistical analysis revealed that defect size seemed to be the critical factor to achieve bone unions [25].

Altogether, it appears that several distinct causes can participate to IMT complications leading to bone non-unions. A strategy that would be helpful to reduce the occurrence of IMT failures would be to gain access to predictive biomarkers of the IM quality at the end of the first IMT step or bone union efficiency during the second Masquelet technique step. Recent research inputs aimed at addressing these issues. By comparing serum levels of matrix metalloproteases (MMPs) in patients responding (bone union achievement) or non-responding (bone union failure) to Masquelet technique, Hambrick et al. [26] demonstrated that MMP-9/MMP-2 ratio was significantly higher in the responder group as compared to the non-responder group, two days after surgery step 1 as well as 4 weeks following surgery step 2. In a similar follow-up, Fisher et al. [27] have shown that serum IGF-1 (insulin-like growth factor-1) cytokine level was significantly increased in IMT responder group as compared to non-responder group from day 2 to week 4 after the second surgery stage. However, IGF-1 levels remained similar 
in both responder and non-responder groups during all the first surgery stage. Thus, both serum MMPs and IGF-1 levels seemed to be potentially interesting predictive biomarkers for IMT outcome in terms of IM quality and bone union achievement.

To conclude, Masquelet technique is a simple surgical procedure not requiring microsurgery skills until the surgeons respect some tips and tricks during both the first and second surgery steps, as fully detailed by Giannoudis et al. [28]. Overall mean rate of successful bone union for large defects (larger than $7 \mathrm{~cm}$ ) using IMT can reach $87 \%$ including revision surgery to overcome bone nonunion resulting from complications. The major drawback of IMT is the early infection risk and control, especially for tibial injury for which soft tissue reconstruction is often required during the first step. To minimize IMT complication occurrence and to complete Giannoudis tips and tricks [28], some important features must be considered:

I. The choice between RIA technique and anterior or posterior iliac crest harvesting seemed to be dictated by the defect size;

II. To expand bone graft volume, mixture of bone substitute with bone graft must not exceed a 1:3 ratio;

III. If the use of antibiotic-coated spacers is common, it can also conceal local infections. It could be interesting to use antibiotic-free spacers as a tool to reveal persistent infection before grafting;

IV. The tremendous importance of rigid fixation in IM procedure: plaster cast must be avoided since it provides unsatisfactory stabilization of the bone defect area; internal fixation by locking plates offers mechanical stability but sometime the device size is inappropriate to the defect location; locked intramedullary nails provide long-lasting stabilization, maintain diaphyseal axis and allow early weight bearing; external fixators afford stable fixation and the possibility to modify bone alignment postoperatively but they are cumbersome $[18,21,29]$.

At last, the choice of the surgical fixation technique (intramedullary nail, locking plates or external fixator) and the way of bone graft harvesting technique (RIA or ICBG) would be optimized depending on the patient needs in terms of size and body location of the bone defects. The availability of predictive biomarkers for IM efficiency would also help to overcome or anticipate some IMT complications leading to bone union failure.

\section{Acknowledgements}

This work was supported by a grant from the Délégation Générale de l'Armement (DGA; Grant SAN-1-0217 to Dr. Marjorie Durand) of the French Ministry of Defense.

\section{References}

1. Baldwin P, DJ Li, DA Auston, HS Mir, RS Yoon, et al. (2019) Autograft, Allograft, and Bone Graft Substitutes: Clinical Evidence and Indications for Use in the Setting of Orthopaedic Trauma Surgery. J Orthop Trauma 33(4): 203-213.
2. Belthur MV, JD Conway, G Jindal, A Ranade, JE Herzenberg (2008) Bone graft harvest using a new intramedullary system. Clin Orthop 466(12): 2973-2980.

3. Cox G, E Jones, D McGonagle, PV Giannoudis (2011) Reamer-irrigatoraspirator indications and clinical results: a systematic review. Int Orthop 35(7): 951-956

4. Weiland AJ, TW Phillips, MA Randolph (1984) Bone grafts: a radiologic, histologic, and biomechanical model comparing autografts, allografts, and free vascularized bone grafts. Plast Reconstr Surg 74(3): 368-379.

5. Masquelet AC, F Fitoussi, T Begue, GP Muller (2000) [Reconstruction of the long bones by the induced membrane and spongy autograft]. Ann Chir Plast Esthet 45(3): 346-353.

6. Wang J, Q Yin, S Gu, Y Wu, Y Rui (2019) Induced membrane technique in the treatment of infectious bone defect: A clinical analysis. Orthop Traumatol Surg Res S1877.

7. Villemagne T, C Bonnard, F Accadbled, M L'kaissi, B de Billy, et al. (2011) Intercalary segmental reconstruction of long bones after malignant bone tumor resection using primary methyl methacrylate cement spacer interposition and secondary bone grafting: the induced membrane technique. J Pediatr Orthop 31(5): 570-576.

8. Taylor BC, BG French, TT Fowler, J Russell, A Poka (2012) Induced membrane technique for reconstruction to manage bone loss. J Am Acad Orthop Surg 20(3): 142-150.

9. Pelissier P, AC Masquelet, R Bareille, SM Pelissier, J Amedee (2004) Induced membranes secrete growth factors including vascular and osteoinductive factors and could stimulate bone regeneration. J Orthop Res Off Publ Orthop Res Soc 22(1): 73-79.

10. Christou C, RA Oliver, Y Yu, WR Walsh (2014) The Masquelet technique for membrane induction and the healing of ovine critical sized segmental defects. PloS One 9(12): e114122.

11. Zwetyenga N, S Catros, A Emparanza, C Deminiere, F Siberchicot, et al. (2009) Mandibular reconstruction using induced membranes with autologous cancellous bone graft and HA-betaTCP: animal model study and preliminary results in patients. Int J Oral Maxillofac Surg 38(12): 1289-1297.

12. Viateau V, G Guillemin, Y Calando, D Logeart, K Oudina, et al. (2006) Induction of a barrier membrane to facilitate reconstruction of massive segmental diaphyseal bone defects: an ovine model. Vet Surg VS 35(5): 445-452.

13. Cuthbert R, S Churchman, H Tan, D McGonagle, E Jones, P Giannoudis (2013) Induced periosteum a complex cellular scaffold for the treatment of large bone defects. 57(2): 484-492.

14. Gouron R, L Petit, C Boudot, I Six, M Brazier, et al. (2017) Osteoclasts and their precursors are present in the induced-membrane during bone reconstruction using the Masquelet technique. J Tissue Eng Regen Med 11(2): 382-389.

15. Luangphakdy V, G Elizabeth Pluhar, NS Piuzzi, J-C D’Alleyrand, CS Carlson et al. (2017) The Effect of Surgical Technique and Spacer Texture on Bone Regeneration: A Caprine Study Using the Masquelet Technique. Clin Orthop 475(10): 2575-2585.

16. El-Alfy BS, AM Ali (2015) Management of segmental skeletal defects by the induced membrane technique. Indian J Orthop 49(6): 643-648.

17. Azi ML, AA de A Teixeira, RB Cotias, A Joeris, M Kfuri (2016) Membrane Induced Osteogenesis in the Management of Posttraumatic Bone Defects. J Orthop Trauma 30(10): 545-550.

18. Aurégan J-C, T Bégué, G Rigoulot, C Glorion, S Pannier (2016) Success rate and risk factors of failure of the induced membrane technique in children: a systematic review. Injury 47 Suppl 6: S62-S67.

19. Morelli I, L Drago, DA George, E Gallazzi, S Scarponi, CL Romanò, et al.(2016) Masquelet technique: myth or reality? A systematic review and meta-analysis. Injury 47 Suppl 6: S68-S76. 
20. Giannoudis PV, PJ Harwood, T Tosounidis, NK Kanakaris (2016) Restoration of long bone defects treated with the induced membrane technique: protocol and outcomes. Injury 47(6): S53-S61.

21. Siboni R, E Joseph, L Blasco, C Barbe, O Bajolet et al. (2018) Management of septic non-union of the tibia by the induced membrane technique. What factors could improve results? Orthop Traumatol Surg Res OTSR 104(6): 911-915.

22. Giotikas D, N Tarazi, L Spalding, M Nabergoj, M Krkovic (2019) Results of the Induced Membrane Technique in the Management of Traumatic Bone Loss in the Lower Limb: A Cohort Study. J Orthop Trauma 33(3): 131-136.

23. Calori GM, M Colombo, EL Mazza, S Mazzola, E Malagoli, GV Mineo et al.(2014) Incidence of donor site morbidity following harvesting from iliac crest or RIA graft. Injury 45(6): S116- S120.

24. Scolaro JA, ML Schenker, S Yannascoli, K Baldwin, S Mehta, et al. (2014) Cigarette smoking increases complications following fracture: a systematic review. J Bone Joint Surg Am 96(8): 674-681.

25. Metsemakers WJ, G Claes, PJ Terryn, A Belmans, H Hoekstra, et al. (2019) Reamer- Irrigator-Aspirator bone graft harvesting for treatment of segmental bone loss: analysis of defect volume as independent risk factor for failure. Eur J Trauma Emerg Surg Off Publ Eur Trauma Soc 45(1): 21-29.

26. Haubruck P, R Heller, P Apitz, A Kammerer, A Alamouti, et al. (2018) Evaluation of matrix metalloproteases as early biomarkers for bone regeneration during the applied Masquelet therapy for non-unions. Injury 49(10): 1732-1738.

27. Fischer C, J Doll, M Tanner, T Bruckner, G Zimmermann, et al. (2016) Quantification of TGF- 31 , PDGF and IGF-1 cytokine expression after fracture treatment vs. non-union therapy via masquelet. Injury $47(2)$ : 342-349.

28. Giannoudis PV, O Faour, T Goff, N Kanakaris, R Dimitriou (2011) Masquelet technique for the treatment of bone defects: tips-tricks and future directions. Injury 42(6): 591-598.

29. Chadayammuri V, M Hake, C Mauffrey (2015) Innovative strategies for the management of long bone infection: a review of the Masquelet technique. Patient Saf Surg 9: 32. 